\title{
Catalytic hairpin assembly combined with graphene oxide for the detection of emetic Bacillus cereus in milk
}

\author{
Zhongxu Zhan, ${ }^{1}$ Bei Yu, ${ }^{1}$ Hui Li, ${ }^{1}$ Leina Yan, ${ }^{1}$ Zoraida P. Aguilar, ${ }^{2}$ and Hengyi $X u^{1 *}$ \\ ${ }^{1}$ State Key Laboratory of Food Science and Technology, Nanchang University, Nanchang, 330047, PR China \\ ${ }^{2}$ Zystein LLC, Fayetteville, AR 72703
}

\begin{abstract}
A fluorescence assay combined with PCR, catalytic hairpin assembly (CHA), and graphene oxide (GO) was established to detect emetic Bacillus cereus in milk samples. The processes of the assay are not new, but components of the processes make the assay useful. Two partially complementary hairpin probes (H1 and FAMH2) were designed according to the target single-strand DNA (ssDNA). The CHA reaction could be initiated only by the target ssDNA, which was generated by the denaturation of PCR amplicons. In the absence of the target ssDNA, CHA reaction could not be triggered, which caused the $\mathrm{H} 1$ and FAM-H2 adsorbing on the surface of GO and exhibiting a low fluorescence intensity. Addition of the target ssDNA resulted in opening of the hairpin $\mathrm{H} 1$ that subsequently hybridized with H2. Then, target ssDNA would be replaced from the $\mathrm{H} 1$ and recycled to promote another $\mathrm{CHA}$ reaction. Through the CHA reaction, multiple $\mathrm{H} 1-\mathrm{H} 2$ duplexes were generated that could not adsorb on the surface of GO. Thus, a strong fluorescence signal would be obtained. The assay showed a limit of detection for emetic B. cereus of $6.2 \times 10^{1} \mathrm{cfu} / \mathrm{mL}$ in pure culture and 5.9 $\times 10^{2} \mathrm{cfu} / \mathrm{mL}$ in spiked milk without enrichment. By changing the PCR primer, the assay developed in this study had potential to detect other bacteria.

Key words: catalytic hairpin assembly, graphene oxide, fluorescence, emetic Bacillus cereus
\end{abstract}

\section{INTRODUCTION}

Bacillus cereus is a gram-positive, rod-shaped, and spore-forming bacilli that can pose significant threats to public health (Priha et al., 2004; Dierick et al., 2005; Fricker et al., 2007). Bacillus cereus can cause

Received October 9, 2018.

Accepted February 3, 2019.

*Corresponding author: kidyxu@163.com or HengyiXu@ncu.edu.cn
2 different gastrointestinal diseases of food poisoning, namely diarrhea and emesis (Arslan et al., 2014; Du et al., 2018; Li et al., 2018). The diarrhea type is caused by heat-labile enterotoxins whereas the emesis type is the result of heat-stable cereulide (Martínez-Blanch et al., 2011). It has been reported that $B$. cereus can be found in food such as pasta, rice, meat, noodles, milk, and other dairy products (Martinez-Blanch et al., 2010; Shiota et al., 2010). Moreover, emetic B. cereus can cause spore germination when foods are boiled, cooled, and stored at room temperature. Considering emetic $B$. cereus poses significant threats to public health (Delbrassinne et al., 2015), a specific and accurate method for detection of emetic B. cereus in food is in demand.

Conventional detection methods for $B$. cereus are time consuming ( -7 d; Madiyar et al., 2015; Porcellato et al., 2016). Polymerase chain reaction has been developed for the rapid and accurate detection of foodborne pathogens (Mao et al., 2016; Luo et al., 2017). However, conventional PCR has been limited by the low resolution of gel electrophoresis. Even worse, the use of ethidium bromide could affect the operator's health (Yu et al., 2018). Real-time PCR provides a higher sensitivity with no additional postamplification; however, real-time PCR needs expensive equipment and fluorescence dyes, and even worse, it is difficult to design the primer and requires trained analysts to operate (Zhou et al., 2017; Hiitiö et al., 2018). Therefore, the development of low-cost and simple methods to make up for these shortcomings is vitally important.

In recent years, a series of detection methods based on fluorescence was established. However, most of the fluorescence strategies required a fluorophore-labeled DNA and the quencher-labeled cDNA or a duallabeled beacon at each end of the DNA (Zheng et al., 2012). On the one hand, it was expensive to synthesize fluorophore-labeled DNA, and on the other hand, the labeled DNA had weak affinity to the target DNA (Liu et al., 2013; Xing et al., 2016). To make up for the disadvantages, researchers have been working on new approaches. 
Table 1. DNA sequences of primers and hairpin probes used in this study

\begin{tabular}{lll}
\hline Nucleic acid & Sequence $\left(5^{\prime}-3^{\prime}\right)$ & Reference \\
\hline cesB-Forward & CTGACGAAGAGATGCGATTGC & Yu et al. (2018) \\
cesB-Reverse & CGTGGTTCACATTCGGAAGG & Yu et al. (2018) \\
H1 & ATGGAAAAGAGAAGATGGCAAAGAAGAAGGTGTTTGCCATCTTCTCT & This study \\
FAM-H2 & FAM-ATGGCAAACACCTTCTTCTTTGCCATCTTCTCTAGAAGAAGGTGTTAGTA & This study \\
Target ssDNA ${ }^{1}(93 \mathrm{nt})$ & CGTGGTTCACATTCGGAAGGCGAAATACTTCTTCATAAGTAATTCCATCTTCTCT & This study \\
& TTTCCATAGCTTTGCTGCAATCGCATCTCTTCGTCAG & \\
\hline
\end{tabular}

${ }^{1} \mathrm{ssDNA}=$ single-strand DNA.

Graphene oxide $(\mathbf{G O})$ is an important derivative of graphene and is well-known for its extraordinary electronic, optical, and thermal properties as well as large surface area, good water dispersibility, and biocompatibility (Jhaveri et al., 2000). Graphene oxide exhibits unique fluorescence-quenching capability when organic fluorescent molecules adsorb on it (Ho and Leclerc, 2004). Also, it has been reported that single-strand DNA (ssDNA) can adsorb onto the surface of GO by $\pi-\pi$ stacking and electrostatic interactions, but hardly interacts with rigid double-stranded DNA (dsDNA) or well-folded structures (Feng et al., 2013). Based on the fluorescence-quenching capability and the affinity difference, a novel bioassay platform for DNA detection through GO fluorescence has been reported. However, a single target DNA usually conjugates only to one fluorescence probe, which limits the sensitivity.

Recently, a series of signal amplification methods was developed; catalytic hairpin assembly (CHA) amplification technique is an enzyme-free amplification technique that relies on hybridization and strand-exchange reactions to achieve amplification (Chen et al., 2016; Li et al., 2016; Dai et al., 2018). The reaction mechanism can be explained as follows: the initiator strands can trigger a pair hairpin to form a duplex, then the initiator strands can be released to the reaction system for the subsequent CHA reaction. Thus, by introducing only one ssDNA, the reaction could produce multiple dsDNA (Zhang et al., 2011; Luan et al., 2017). Furthermore, CHA is a sequence-dependent reaction, and thus, it ensures specificity.

Based on the above considerations, we developed a novel and enzyme-free assay based on catalyzed hairpin DNA assembly combined with GO to detect emetic $B$. cereus. In this assay, the target ssDNA was generated by PCR amplicons and the hairpin probes opened only after binding with the target ssDNA. In the absence of the target ssDNA, hairpin probes were adsorbed on the surface of GO, resulting in fluorescence quenching. Upon the addition of the target ssDNA, a cascade of dsDNA between the 2 hairpin probes was formed, which had low affinity with GO, and then the hairpin probes would separate from the GO and a strong fluorescence signal would be obtained. The proposed method could be a cost-effective and label-free fluorescence system. By changing the PCR primer, the assay developed in this study had potential to detect other bacteria.

\section{MATERIALS AND METHODS}

\section{Reagents and Materials}

Luria-Bertani medium was purchased from Land Bridge Technology Co. Ltd. (Beijing, China). The GO was bought from XFNANO Materials Technology Co. Ltd. (Nanjing, China). The TaKaRa MiniBest DNA Fragment Purification Kit was purchased from TaKaRa Biotech Co. Ltd. (Dalian, China). Two $\times$ Taq master mix was purchased from Tiangen Biotech Co., Ltd. (Beijing, China). HEPES buffer (25 mM HEPES; 200 $\mathrm{m} M \mathrm{KCl} ; 200 \mathrm{~m} M \mathrm{NaCl} ; 1 \%$ DMSO; pH 7.4) was used in the experiments. The oligonucleotides (Table 1), including specific primers, hairpin structure H1, FAM modified hairpin structure $\mathrm{H} 2$, and the target ssDNA (93 nt), were synthesized by TsingKe Biotech. Co. Ltd. (Beijing, China). To ensure the formation of hairpin structure, the hairpin structure oligonucleotides were heated at $95^{\circ} \mathrm{C}$ for $5 \mathrm{~min}$ and then cooled to room temperature.

\section{Bacterial Culture}

The bacteria used in this experiment were listed in Table 2. All bacterial strains were cultured in LuriaBertani broth overnight at $37^{\circ} \mathrm{C}$ with continuous shaking (180 rpm). Sterile PBS (0.01 $M$, pH 7.4) was applied to suspend fresh target bacteria to obtain 10 serial dilutions. The counts of bacterial colonies were determined using the conventional plate counting method at $37^{\circ} \mathrm{C}$ for $18 \mathrm{~h}$.

\section{DNA Extraction and PCR Conditions}

The genomic DNA for all the bacteria were harvested using the boiling method. One milliliter of the bacteria 
was washed twice in an equal volume of bacteria-free PBS and resuspended in $50 \mu \mathrm{L}$ of sterilized water, then boiled in a water bath for 25 min. After centrifugation at $12,000 \times g$ for 5 min at room temperature, the DNA in supernatant was used as template for PCR.

The specific primers used in this study were listed in Table 1. The PCR amplification was carried out in a total reaction volume of $50 \mu \mathrm{L}$ containing $25 \mu \mathrm{L}$ of 2 $\times$ Taq Master Mix, $2 \mu \mathrm{L}$ of $10 \mu M$ each forward and reverse ces $B$ primer, $2 \mu \mathrm{L}$ of genomic DNA, and $19 \mu \mathrm{L}$ of $\mathrm{dH}_{2} \mathrm{O}$. The PCR conditions were as follows: $94^{\circ} \mathrm{C}$ for 5 min, followed by 30 cycles of denaturation at $94^{\circ} \mathrm{C}$ for $30 \mathrm{~s}$, annealing at $60^{\circ} \mathrm{C}$ for $30 \mathrm{~s}$, and elongation at $72^{\circ} \mathrm{C}$ for $30 \mathrm{~s}$, with a final elongation at $72^{\circ} \mathrm{C}$ for $10 \mathrm{~min}$.

\section{Purification and Denaturation}

After the PCR, the PCR amplicons were subjected to purification and denaturation. The PCR amplicons were purified according to the operation steps of the DNA Fragment Purification Kit. After purification, the PCR amplicons were put into the PCR apparatus and denatured at $94^{\circ} \mathrm{C}$ for $5 \mathrm{~min}$. Then the denatured amplicons were quickly transferred to ice water to obtain the target ssDNA.

\section{Agarose Gel Electrophoresis}

Agarose gel electrophoresis was used to test feasibility of the catalyzed hairpin assembly. The H1, H2, mixture 1 (target ssDNA and H1), mixture 2 (H1 and $\mathrm{H} 2$ ), and mixture 3 (H1, H2, and target ssDNA) were analyzed and the final concentration of all the DNA was $1 \mu M$. After incubation at $37^{\circ} \mathrm{C}$ for $30 \mathrm{~min}$, the test samples were added into $4 \%$ agarose gel. The gel was run under a constant voltage of $80 \mathrm{~V}$ for $45 \mathrm{~min}$

Table 2. Target and nontarget bacteria strains used in this study

\begin{tabular}{lll}
\hline Strain of bacteria & Strain ID & Source $^{1}$ \\
\hline Bacillus cereus $(\mathrm{n}=5)$ & JDZ0102Y & JX-CDC \\
& PZ0063L & JX-CDC \\
& JA0064 & JX-CDC \\
& JX0121LY & JX-CDC \\
Staphylococcus aureus & FX0106Y & JX-CDC \\
Bacillus subtilis & 26001 & CMCC \\
Cronobacter sakazakii & 63501 & CMCC \\
Salmonella Paratyphi B & 45401 & CMCC \\
Listeria monocytogenes & 50049 & JX-CDC \\
\hline
\end{tabular}

${ }^{1} \mathrm{ATCC}=$ American Type Culture Collection; $\mathrm{CMCC}=$ China Medical Culture Collection; JX-CDC $=$ Jiang Xi Province Center for Disease Control and Prevention. followed by imaging under the UV trans illuminator (Bio-Rad, Hercules, CA).

\section{GO-Based CHA Reaction and Fluorescence Detection}

The H1, FAM-H2, and denatured PCR amplicons were mixed in HEPES buffer, followed by incubation at $37^{\circ} \mathrm{C}$ for $30 \mathrm{~min}$. Subsequently, GO was added to the mixture and incubated $10 \mathrm{~min}$ at room temperature. Finally, the reaction mixture was transferred to 96well plate for fluorescence detection. The fluorescence intensity was monitored using a multifunctional microplate reader (Thermo Fisher Scientific, Waltham, MA) by exciting the sample at $488 \mathrm{~nm}$ and measuring the emission at $520 \mathrm{~nm}$. The slits for excitation and emission were both set at $5 \mathrm{~nm}$. All the experiments were repeated in triplicate.

\section{Detection of Emetic B. cereus Using Conventional PCR}

The practicability of the conventional PCR assay was evaluated using the emetic $B$. cereus in pure culture. One milliliter of emetic $B$. cereus in pure culture was centrifuged at $12,000 \times g$ for $5 \mathrm{~min}$ at room temperature and resuspended in an equal volume of sterile water. Then the emetic B. cereus was diluted with sterile water ranging from $6.2 \times 10^{0}$ to $6.2 \times 10^{7} \mathrm{cfu} / \mathrm{mL}$. Next, DNA templates were obtained using the boiling method as mentioned above, followed by the PCR amplification. Finally, the PCR amplicons were added into a $2 \%$ agarose gel electrophoresis, run under a constant voltage of $150 \mathrm{~V}$ for $20 \mathrm{~min}$, followed by imaging under the UV trans illuminator (Bio-Rad).

\section{Process of Emetic B. cereus Detection in Artificially Inoculated Milk Samples}

The milk purchased from a local supermarket used in this study was verified for the absence of $B$. cereus by standard culture assays. The milk matrix was obtained by mixing $1 \mathrm{~mL}$ of milk with $9 \mathrm{~mL}$ of sterile PBS. Then a $900-\mu \mathrm{L}$ milk matrix was contaminated with $100 \mu \mathrm{L}$ of the different concentrations of emetic $B$. cereus to obtain a final concentration of $5.9 \times 10^{8}$ to $5.9 \times 10^{1}$ $\mathrm{cfu} / \mathrm{mL}$. These were centrifuged at $8,000 \times g$ for $3 \mathrm{~min}$ at room temperature to remove the large debris. After extracting genomic DNA for PCR, the purified and denatured PCR amplicons were used in a fluorescence detection method. All of the experiments were repeated in triplicate. 


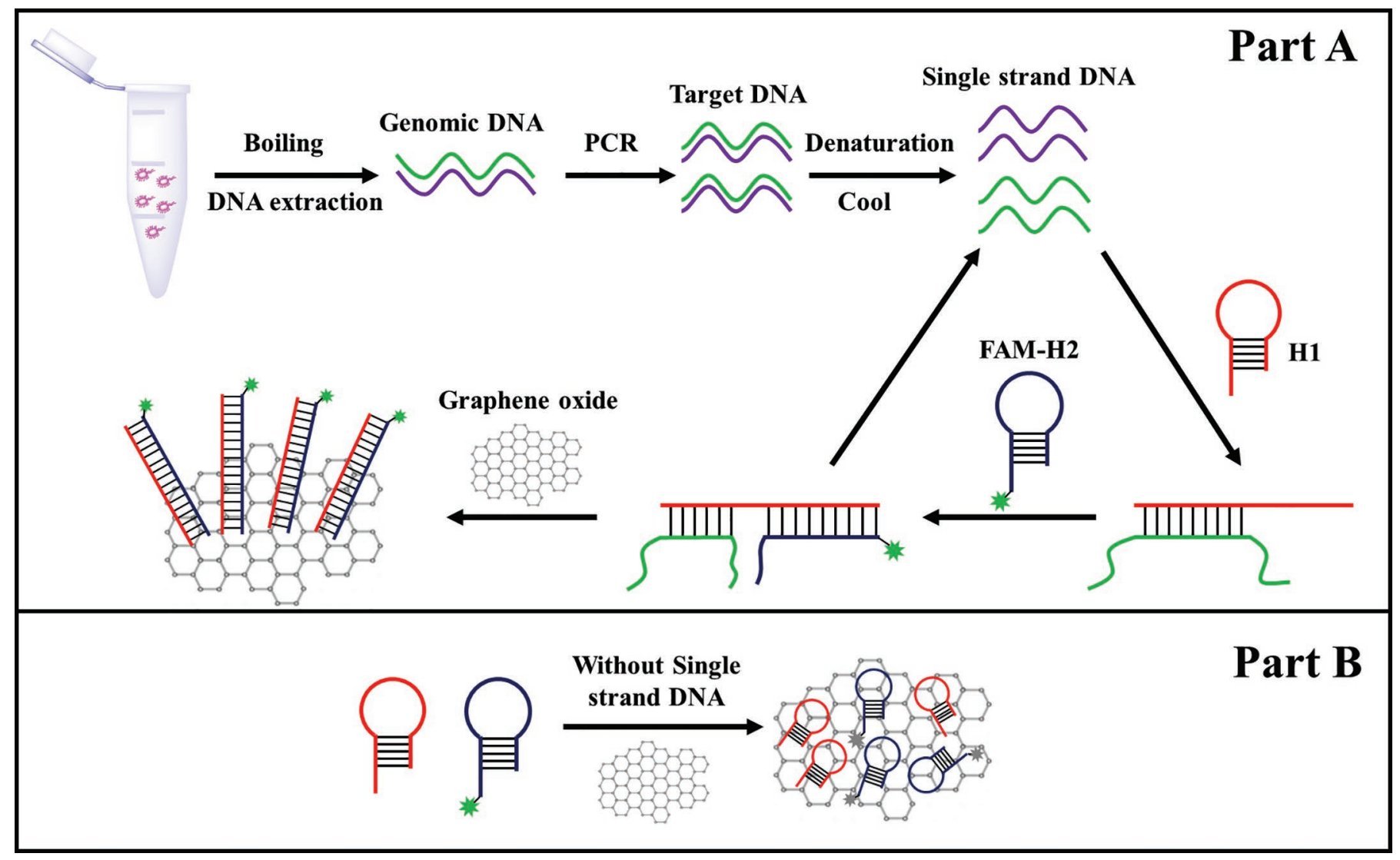

Figure 1. The principle of catalytic hairpin assembly-graphene oxide assay to detect emetic Bacillus cereus.

\section{RESULTS AND DISCUSSION}

\section{Principle of Emetic B. cereus Detection}

The principle of the fluorescence assay is shown in Figure 1 . The 2 partially complementary hairpin probes were designed according to the target ssDNA. The H1 included recognition sequence of the target ssDNA and the FAM-H2. The FAM-H2 included the recognition sequence of $\mathrm{H} 1$ and partly the same sequence as the target ssDNA. First, we used the boiling method to harvest the genomic DNA. Then PCR was used to obtain the target DNA. After PCR amplification, the PCR amplicons were subjected to purification. Then the target ssDNA was obtained by denaturing the PCR amplicons at $94^{\circ} \mathrm{C}$ for $5 \mathrm{~min}$ and quickly transferring them to ice water. Finally, the target ssDNA was used for the fluorescence detection. Part A shows the fluorescence assays with target ssDNA. When the system contained target ssDNA, the H1 would hybridize with target ssDNA and expose the recognition sequence of H2. Subsequently, $\mathrm{H} 2$ would hybridize with the recognition sequence of $\mathrm{H} 1$ and replace the target ssDNA to generate the H1-H2 duplexes. The released target ssDNA would recycle to promote another CHA reaction. Thus, by introducing the target ssDNA, multiple H1-H2 duplexes could be generated that could not absorb on the surface of GO, then a strong fluorescence signal would be obtained. However, in the absence of the target ssDNA (part B), 2 hairpin probes could not hybridize spontaneously because the complementary sequences were locked in their stem. Thus, the sticky ends of the hairpin probe would adsorb on the GO surface by $\pi-\pi$ interaction, which resulted in low fluorescence intensity. Therefore, the concentration of the bacteria could be quantified based on the intensity of the fluorescence signal. Thus, the complete time for the detection of emetic B. cereus including the time for sample preparation, PCR amplification, purification, and denaturation, and CHA amplification would be about $3.5 \mathrm{~h}$.

\section{Feasibility of This Method}

To demonstrate the viability of the proposed strategy, the fluorescence signals of the system containing differ- 
A
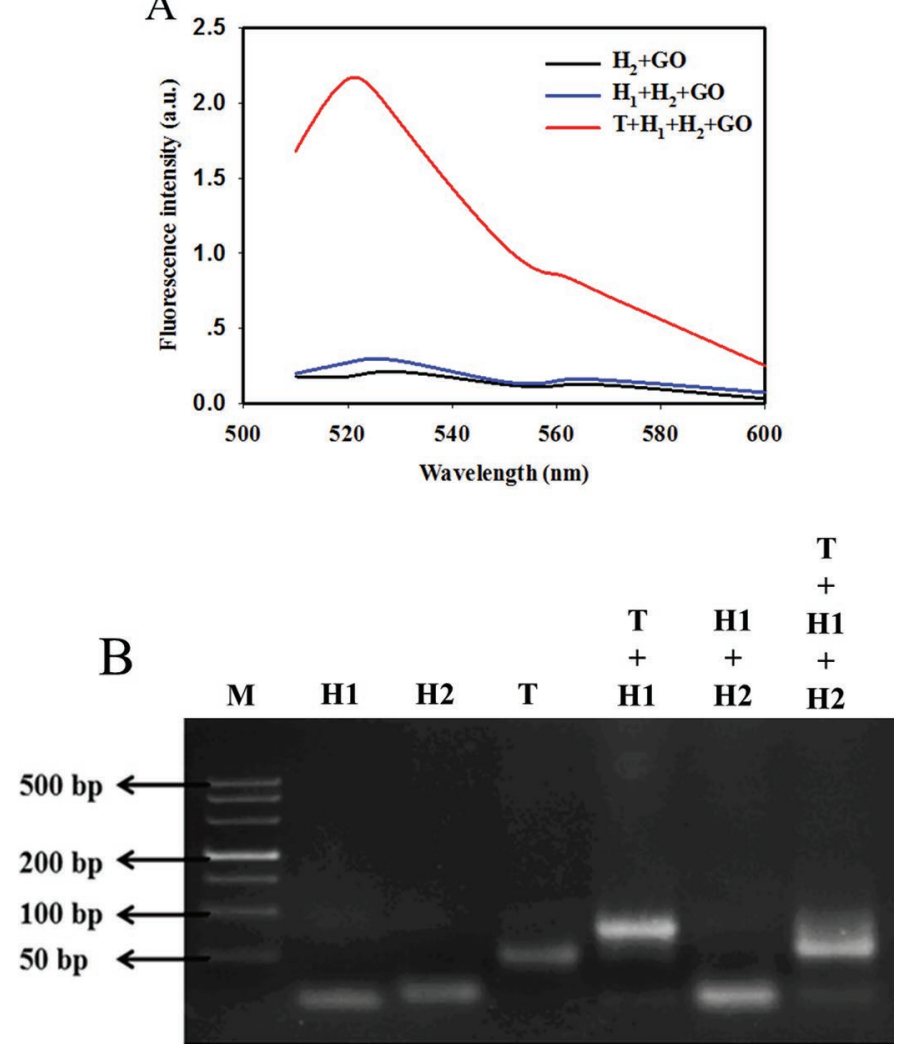

Figure 2. (A) Fluorescence spectra of different reactants: (1) H2 + graphene oxide (GO), (2) H1 + H2 + GO, and (3) $\mathrm{T}+\mathrm{H} 1+$ $\mathrm{H} 2+\mathrm{GO}$; (B) 4\% gel electrophoresis results of the proposed system under the different conditions. $\mathrm{M}=\mathrm{DL} 500$ marker, and $\mathrm{T}=$ target single-strand DNA. All of the tests were repeated in triplicate. a.u. = arbitrary units.

ent conditions were shown in Figure 2A. The system without target ssDNA exhibited very weak fluorescence intensity. Upon the addition of the target ssDNA, the fluorescence intensity increased significantly due to the formation of the H1-H2 duplexes though the CHA reaction. To determine the occurrence of $\mathrm{CHA}$ reaction, $4 \%$ agarose gel electrophoresis analysis was performed. As shown in Figure 2B, when H1 mixed with target ssDNA in lane 4, a new brighter band appeared. This indicated the target ssDNA could open the $\mathrm{H} 1$ and produced target ssDNA-H1 duplexes. When the H1 and $\mathrm{H} 2$ were mixed, there were no new bands in lane 5, indicating that the $\mathrm{H} 1$ and $\mathrm{H} 2$ did not react spontaneously with each other. When the H1, H2, and target ssDNA were mixed, there were new bands in lane 6 . This suggested that the target ssDNA initiated a CHA reaction and generated the $\mathrm{H} 1-\mathrm{H} 2$ duplexes. These 2 results demonstrated the target ssDNA could catalyze the hybridization of $\mathrm{H} 1, \mathrm{H} 2$ and subsequently generated the H1-H2 duplexes. All the experiments were repeated in triplicate.

\section{Optimization of Experimental Parameters}

To obtain better performance of emetic $B$. cereus detection, the dosage of GO, incubation time, and CHA reaction time were carefully optimized. As GO acted as a quencher, it was important to optimize the concentration of GO and the incubation time. If the GO concentration was low, it could cause a strong background signal. Also, if the GO concentration was too high, it could cause a negative signal. Thus, a suitable concentration of GO was vitally important to the assay. In this study, we used $3 \mathrm{n} M \mathrm{H} 1 / \mathrm{H} 2$ and $50 \mu \mathrm{g} / \mathrm{mL}$ of GO to study the quenching effect. The signal to noise ratio $\mathrm{F} / \mathrm{F}_{0}$ ( $\mathrm{F}$ and $\mathrm{F}_{0}$ represented the reaction system with and without the target ssDNA, respectively) was used to investigate the quenching effect. As shown in Figure $3 \mathrm{~A}$, as the volume of GO increased from 0 to 10 $\mu \mathrm{L}$, the $\mathrm{F} / \mathrm{F}_{0}$ also increased. When the volume reached $10 \mu \mathrm{L}$, it recorded the highest signal. Therefore, the optimized volume of $50 \mu \mathrm{g} / \mathrm{mL}$ of GO was $10 \mu \mathrm{L}$.

The effects of CHA reaction time and GO incubation time were also investigated. As shown in Figure 3B, the $\mathrm{F} / \mathrm{F}_{0}$ increased with the $\mathrm{CHA}$ reaction time from 0 to $30 \mathrm{~min}$, then decreased after $30 \mathrm{~min}$; thus, the optimal CHA reaction time was 30 min. Figure $3 \mathrm{C}$ showed the fluorescence intensity changed with the incubation time from 0 to 22 min after the addition of GO. It also showed the fluorescence intensity of the negative control group decreased rapidly and then stabilized. However, the fluorescence intensity of the positive group after the addition of GO decreased slightly and then stabilized. Thus, 12 min was adopted as the optimal incubation time of the GO for subsequent studies. All of the experiments were repeated in triplicate.

\section{Application of the CHA-GO Method for Detection of Emetic B. cereus in Pure Culture}

To demonstrate the sensitivity of the developed method, different concentrations of emetic $B$. cereus were measured under the optimal experimental conditions. Figure 4 shown the fluorescence intensity changed with the concentrations of emetic $B$. cereus. As the concentration of emetic $B$. cereus increased, the fluorescence intensity was also increasing. A linear regression equation between the concentration of the emetic $B$. cereus and the fluorescence intensity was given by $\mathrm{y}=0.3427 \mathrm{x}$ $+0.1534\left(R^{2}=0.9857\right)$, where y was the fluorescence intensity at $520 \mathrm{~nm}$ and $\mathrm{x}$ was the concentration of emetic B. cereus that ranged from $6.2 \times 10^{0}$ to 6.2 $\times 10^{7} \mathrm{cfu} / \mathrm{mL}$. Based on the cut-off [negative control (NC) + 3 SD; Xu et al., 2017], the sensitivity of the method in pure culture was $6.2 \times 10^{1} \mathrm{cfu} / \mathrm{mL}$. All of the experiments were repeated in triplicate. 
A

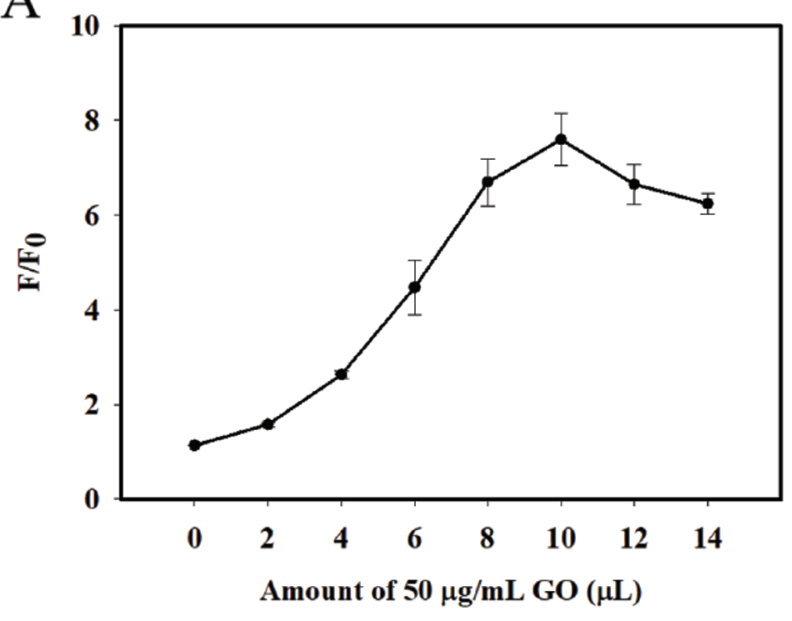

B
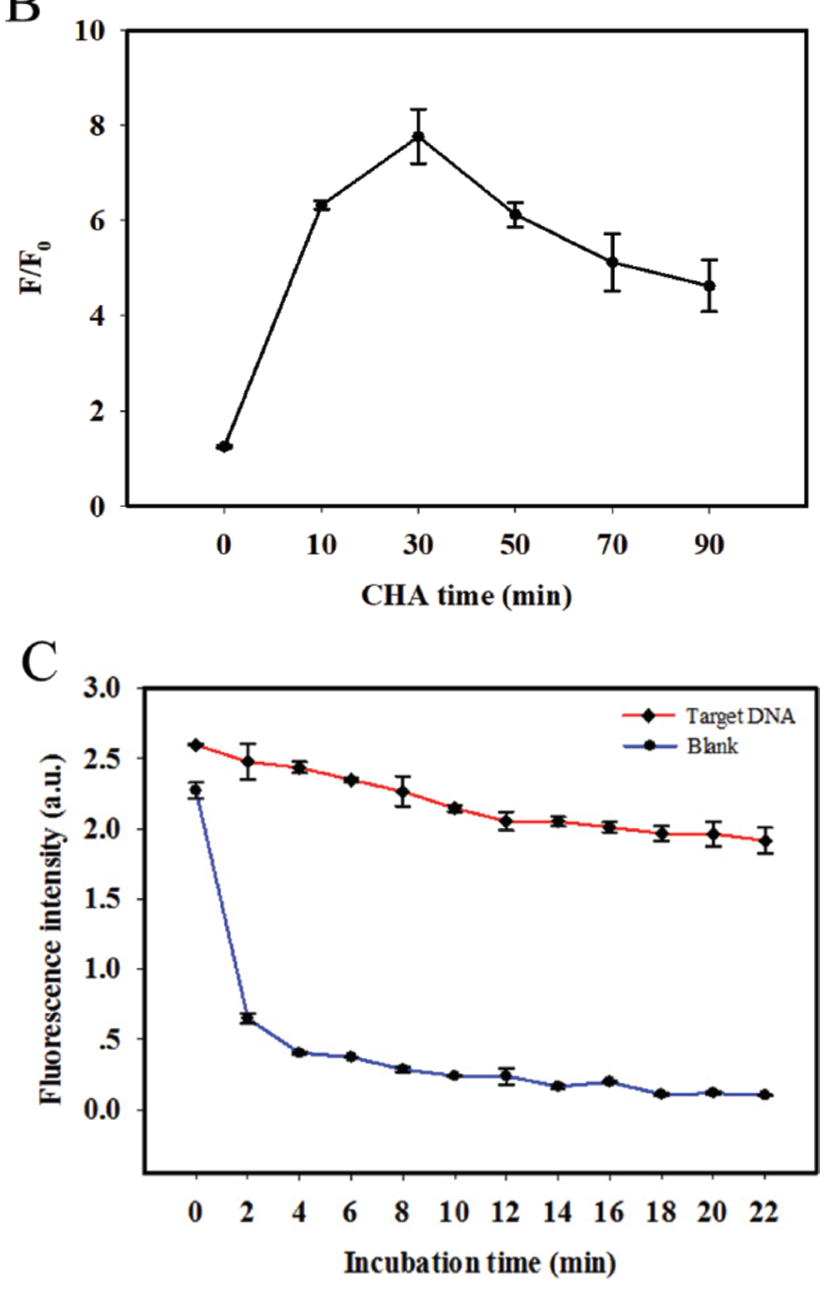

Figure 3. (A) Optimization of graphene oxide (GO) volume, (B) optimization of catalytic hairpin assembly $(\mathrm{CHA})$ reaction time, and (C) fluorescence intensity (a.u. = arbitrary units) under different incubation times of GO and CHA products. All of the tests were repeated in triplicate; each error bar represents $\mathrm{SD}$. The $\mathrm{F} / \mathrm{F}_{0}$ represent the fluorescence intensity by CHA reaction with target single-strand DNA (ssDNA)/the fluorescence intensity by CHA reaction without target ssDNA.

\section{Specificity of the CHA-GO Assay in Pure Culture}

The specificity of the proposed assay was evaluated by selecting 8 strains of nontarget bacteria (Table 2 ). As shown in Figure 5, the nontarget bacteria (approximately $10^{7} \mathrm{cfu} / \mathrm{mL}$ ) containing Bacillus subtilis, Listeria monocytogenes, Salmonella Paratyphi, Staphylococcus aureus, Cronobacter sakazakii, and non-emetic $B$. cereus showed low fluorescence intensity. However, a high fluorescence intensity was obtained using the emetic B. cereus. This suggested that this fluorescence method had a high specificity to distinguish between target bacteria and other bacteria. All of the experiments were repeated in triplicate.

\section{Detection of Emetic B. cereus Using Conventional PCR}

The ability of the conventional PCR assay was also evaluated using the emetic $B$. cereus in pure culture and the limit of detection (LOD) was established. The concentrations of emetic B. cereus ranged from $6.2 \times$ $10^{7}$ to $6.2 \times 10^{0} \mathrm{cfu} / \mathrm{mL}$. As shown in Figure 6 , the $\mathrm{NC}$ lane (the sterile water was used as the template for PCR) had no target DNA band, and when the concentration of emetic B. cereus was below the $10^{4} \mathrm{cfu} / \mathrm{mL}$, no target DNA band was exhibited on the gel. Thus, the LOD of the traditional PCR assay for the detection of emetic B. cereus in pure culture was $6.2 \times 10^{4} \mathrm{cfu} /$ $\mathrm{mL}$. All of the experiments were repeated in triplicate.

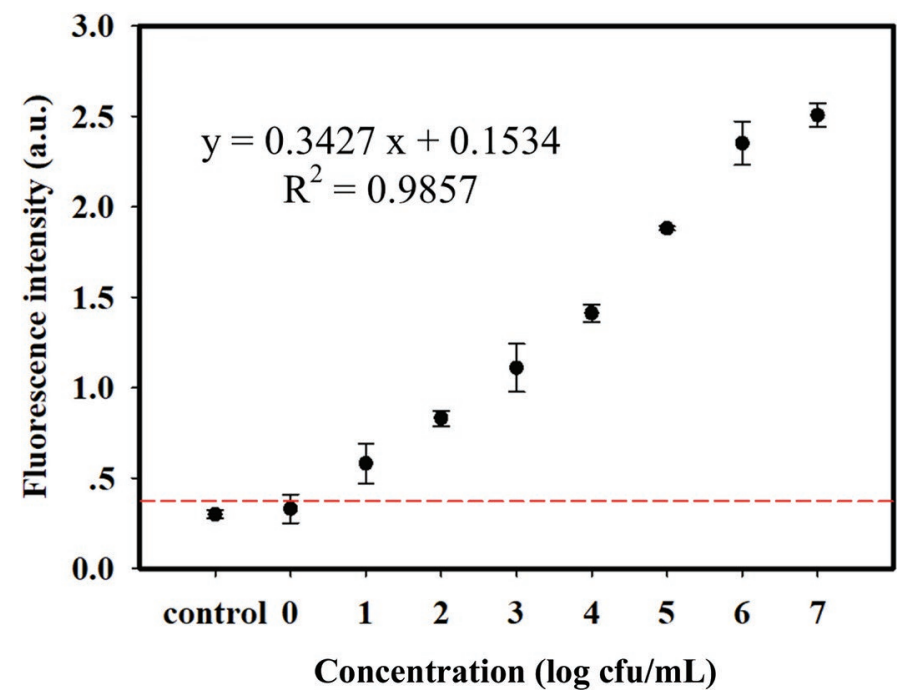

Figure 4. The limit of detection (LOD) of the catalytic hairpin assembly (CHA)-graphene oxide (GO) assay for emetic Bacillus cereus in pure culture. All of the tests were repeated in triplicate. a.u. $=$ arbitrary units. Each error bar represents SD, and the dashed line represents the cut-off [negative control $(\mathrm{NC})+3 \mathrm{SD}$ ]. 


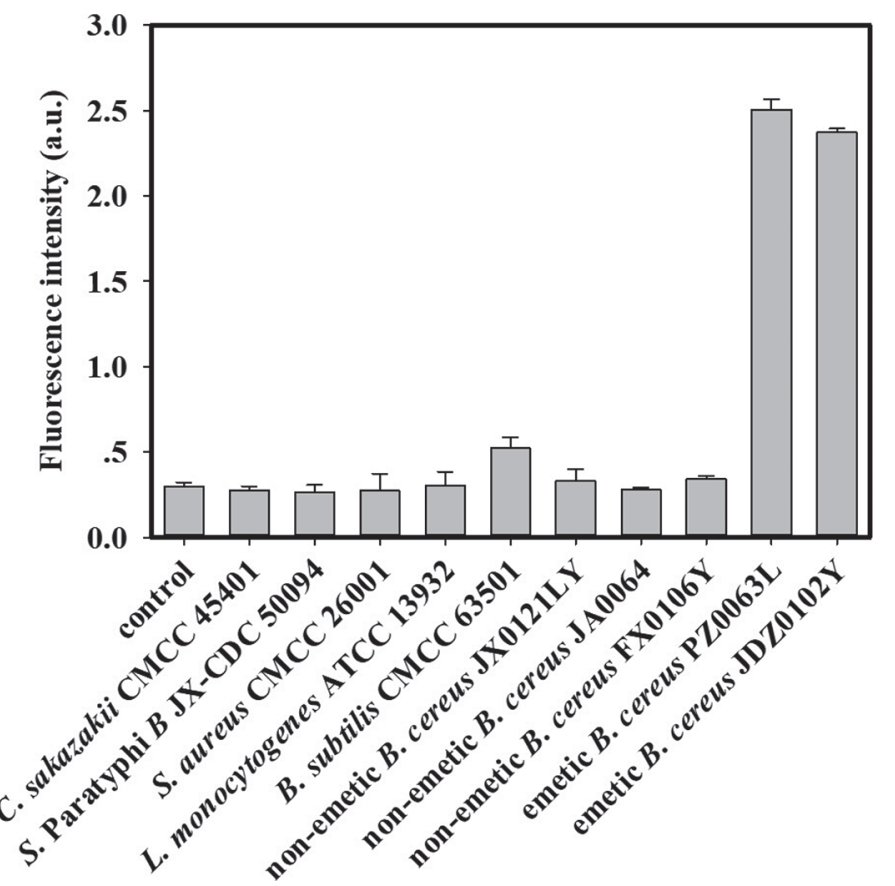

Figure 5. The specificity of the catalytic hairpin assembly (CHA)graphene oxide (GO) assay for detection of emetic Bacillus cereus in pure culture. All of the tests were repeated in triplicate. a.u. $=$ arbitrary units. ATCC $=$ American Type Culture Collection; $\mathrm{CMCC}$ $=$ China Medical Culture Collection; JX-CDC $=$ Jiang Xi Province Center for Disease Control and Prevention. Error bars represent SD.

\section{Application of the CHA-GO Method for Detection of Emetic B. cereus in Artificially Inoculated Milk Samples}

To verify the practical application, the proposed method was assessed in artificially inoculated milk samples. The results showed (Figure 7) that the fluorescence intensity increased with the concentration of emetic B. cereus from $5.9 \times 10^{1}$ to $5.9 \times 10^{8} \mathrm{cfu} / \mathrm{mL}$. A linear relationship between spiked emetic $B$. cereus from $10^{2}$ to $10^{8} \mathrm{cfu} / \mathrm{mL}$ and fluorescence intensity was

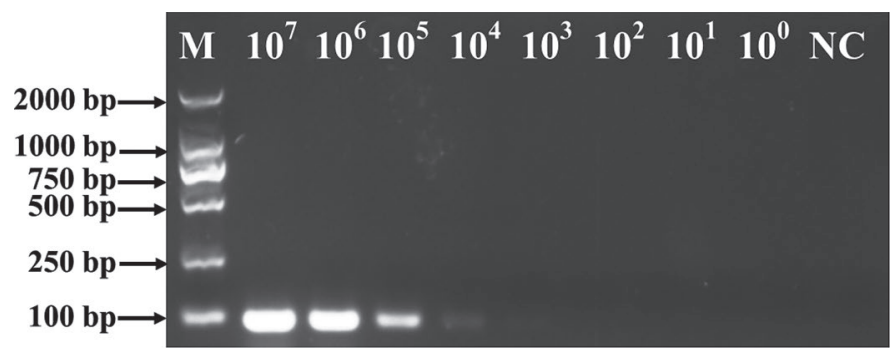

Figure 6. The limit of detection of the conventional PCR assay using 10-fold serial dilution of emetic Bacillus cereus in pure culture from $6.2 \times 10^{7}$ to $10^{0} \mathrm{cfu} / \mathrm{mL}$. Lane $\mathrm{M}=\mathrm{DL} 2000$ marker; lane $\mathrm{NC}=$ negative control. All of the tests were repeated in triplicate. given by $\mathrm{y}=0.2986 \mathrm{x}-0.196\left(\mathrm{R}^{2}=0.9684\right)$, where $\mathrm{y}$ was the fluorescence intensity at $520 \mathrm{~nm}$ and $\mathrm{x}$ was the concentrations of spiked emetic B. cereus). Based on $\mathrm{NC}+3 \mathrm{SD}$, the LOD in spiked milk samples was 5.9 $\times 10^{2} \mathrm{cfu} / \mathrm{mL}$. All of the experiments were repeated in triplicate.

\section{Specificity of the CHA-GO in Artificially Inoculated Milk Samples}

The specificity of the proposed assay was also evaluated in artificially inoculated milk samples. The groups of bacteria $\left(10^{7} \mathrm{cfu} / \mathrm{mL}\right.$ in the milk sample) were used to evaluate the specificity of the assay. Figure 8 showed that only in the presence of emetic $B$. cereus, a high fluorescence intensity would be obtained. This result indicated that the CHA-GO method had a high specificity to distinguish target bacteria or other bacteria in an artificially inoculated milk sample. All of the experiments were repeated in triplicate.

\section{CONCLUSIONS}

Recently, a series of methods for detection of foodborne pathogens has been established. However, these methods are time-consuming and have a high detection limit. In this study, an enzyme-free fluorescence platform for emetic $B$. cereus detection was developed. Fluorescence intensity was restored though CHA reaction, which was initiated by denatured PCR amplicons. The

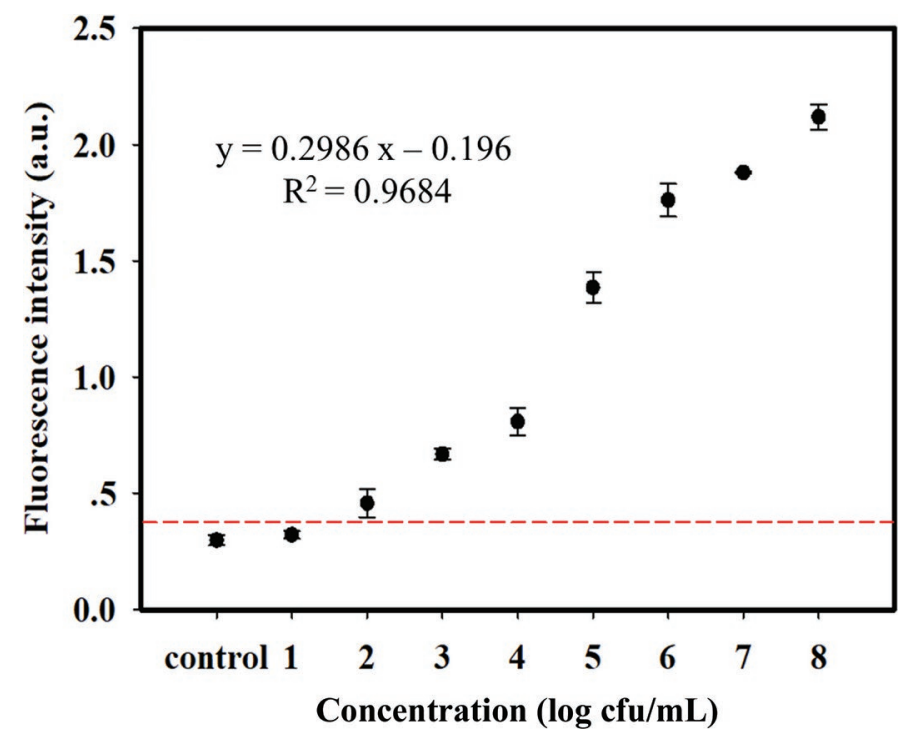

Figure 7. The limit of detection (LOD) of the catalytic hairpin assembly (CHA)-graphene oxide (GO) assay on spiked milk with different concentrations of emetic Bacillus cereus. All of the tests were repeated in triplicate. a.u. = arbitrary units. Error bars represent SD. 


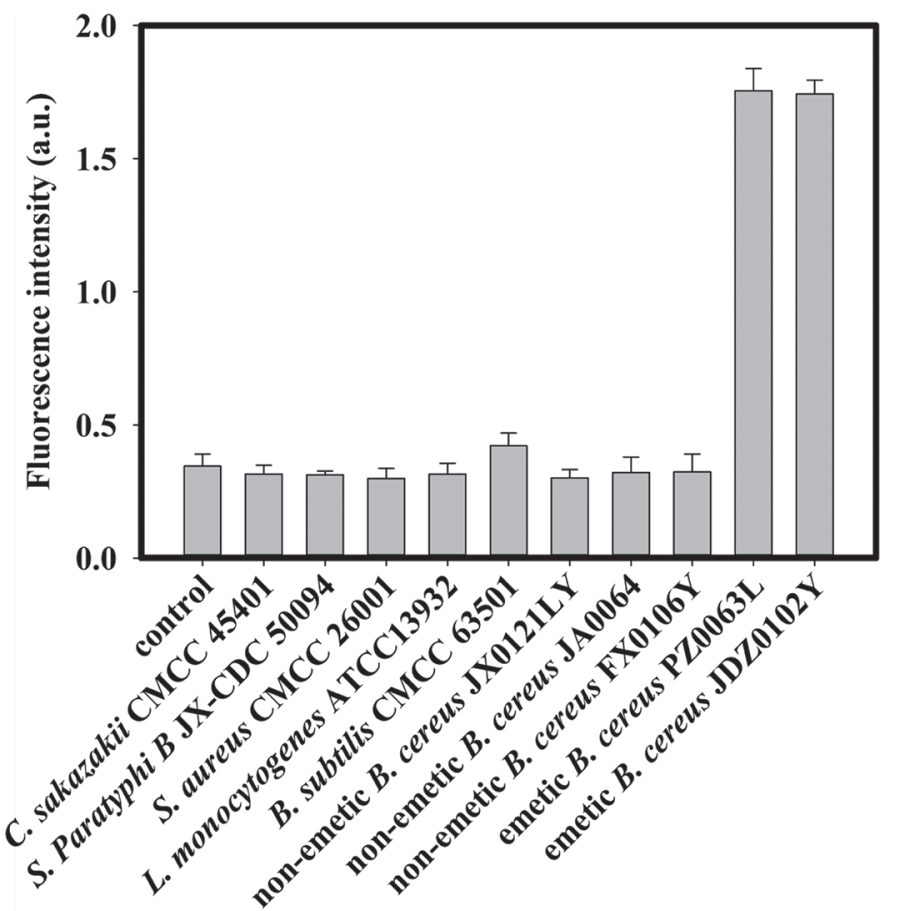

Figure 8. The specificity of the catalytic hairpin assembly (CHA)graphene oxide (GO) assay for detection of emetic Bacillus cereus in artificially inoculated milk samples. All of the tests were repeated in triplicate. a.u. $=$ arbitrary units. ATCC $=$ American Type Culture Collection; $\mathrm{CMCC}=$ China Medical Culture Collection; JX-CDC = Jiang Xi Province Center for Disease Control and Prevention. Error bars represent SD.

hairpin probes $\mathrm{H} 1 / \mathrm{H} 2$ designed in this study achieved the specific detection of emetic $B$. cereus. Under optimal conditions, the CHA-GO method resulted in an LOD of $6.2 \times 10^{1} \mathrm{cfu} / \mathrm{mL}$ in pure culture and $5.9 \times$ $10^{2} \mathrm{cfu} / \mathrm{mL}$ in spiked milk, respectively. Although the method described in this manuscript is relatively timeconsuming and costly, it provides an effective solution to apply CHA to the detection of pathogens, and a relatively sensitive result has been obtained. This work would be interesting to readers of the analysis of other food/dairy products, and by changing the PCR primer, the work has the potential to detect other bacteria.

\section{ACKNOWLEDGMENTS}

The work was supported by the Research Foundation from State Key Laboratory of Food Science and Technology, Nanchang University, China (no. SKLFZZB-201720).

\section{REFERENCES}

Arslan, S., A. Eyi, and R. Küçüksarı. 2014. Toxigenic genes, spoilage potential, and antimicrobial resistance of Bacillus cereus group strains from ice cream. Anaerobe 25:42-46.
Chen, C., N. Li, J. Lan, X. Ji, and Z. He. 2016. A label-free colorimetric platform for DNA via target-catalyzed hairpin assembly and the peroxidase-like catalytic of graphene/Au-NPs hybrids. Anal. Chim. Acta 902:154-159.

Dai, J., Z. Duan, M. Cao, M. Hao, H. He, and D. Xiao. 2018. Rapid DNA detection based on self-replicating catalyzed hairpin assembly using nucleotide base analog pyrrolo-deoxycytidine as fluorophore. Talanta 181:142-146.

Delbrassinne, L., N. Botteldoorn, M. Andjelkovic, K. Dierick, and S. Denayer. 2015. An emetic Bacillus cereus outbreak in a kindergarten: Detection and quantification of critical levels of cereulide toxin. Foodborne Pathog. Dis. 12:84-87.

Dierick, K., E. Van Coillie, I. Swiecicka, G. Meyfroidt, H. Devlieger, A. Meulemans, G. Hoedemaekers, L. Fourie, M. Heyndrickx, and J. Mahillon. 2005. Fatal family outbreak of Bacillus cereus-associated food poisoning. J. Clin. Microbiol. 43:4277-4279.

Du, H., J. Yang, X. Lu, Z. Lu, X. Bie, H. Zhao, C. Zhang, and F Lu. 2018. Purification, characterization, and mode of action of Plantaricin GZ1-27, a novel bacteriocin against Bacillus cereus. J. Agric. Food Chem. 66:4716-4724.

Feng, B., L. Guo, L. Wang, F. Li, J. Lu, J. Gao, C. Fan, and Q. Huang. 2013. A graphene oxide-based fluorescent biosensor for the analysis of peptide-receptor interactions and imaging in somatostatin receptor subtype 2 overexpressed tumor cells. Anal. Chem. 85:7732-7737.

Fricker, M., U. Messelhäußer, U. Busch, S. Scherer, and M. EhlingSchulz. 2007. Diagnostic real-time PCR assays for the detection of emetic Bacillus cereus strains in foods and recent food-borne outbreaks. Appl. Environ. Microbiol. 73:1892-1898.

Hiitiö, H., S. Pyörälä, S. Taponen, P. R. Schultz, and H. Simojoki. 2018. Elimination of experimentally induced bovine intramammary infection assessed by multiplex real-time PCR and bacterial culture. J. Dairy Sci. 101:5267-5276.

Ho, H. A., and M. Leclerc. 2004. Optical sensors based on hybrid aptamer/conjugated polymer complexes. J. Am. Chem. Soc. 126:1384-1387.

Jhaveri, S., M. Rajendran, and A. D. Ellington. 2000. In vitro selection of signaling aptamers. Nat. Biotechnol. 18:1293-1297.

Li, F., F. Li, G. Yang, Z. P. Aguilar, W. Lai, and H. Xu. 2018. Asymmetric polymerase chain assay combined with propidium monoazide treatment and unmodified gold nanoparticles for colorimetric detection of viable emetic Bacillus cereus in milk. Sens. Actuator B-Chem. 255:1455-1461.

Li, X., W. Cheng, D. Li, J. Wu, X. Ding, Q. Cheng, and S. Ding. 2016. A novel surface plasmon resonance biosensor for enzyme-free and highly sensitive detection of microRNA based on multi component nucleic acid enzyme (MNAzyme)-mediated catalyzed hairpin assembly. Biosens. Bioelectron. 80:98-104.

Liu, S., Y. Wang, J. Ming, Y. Lin, C. Cheng, and F. Li. 2013. Enzymefree and ultrasensitive electrochemical detection of nucleic acids by target catalyzed hairpin assembly followed with hybridization chain reaction. Biosens. Bioelectron. 49:472-477.

Luan, Q., N. Gan, Y. Cao, and T. Li. 2017. Mimicking an enzymebased colorimetric aptasensor for antibiotic residue detection in milk combining magnetic loop-DNA probes and CHA-assisted target recycling amplification. J. Agric. Food Chem. 65:5731-5740.

Luo, D., X. Huang, Y. Mao, C. Chen, F. Li, H. Xu, and Y. Xiong. 2017. Two-step large-volume magnetic separation combined with PCR assay for sensitive detection of Listeria monocytogenes in pasteurized milk. J. Dairy Sci. 100:7883-7890.

Madiyar, F. R., S. Bhana, L. Z. Swisher, C. T. Culbertson, X. Huang, and J. Li. 2015. Integration of a nanostructured dielectrophoretic device and a surface-enhanced raman probe for highly sensitive rapid bacteria detection. Nanoscale 7:3726-3736.

Mao, Y., X. Huang, S. Xiong, H. Xu, Z. P. Aguilar, and Y. Xiong 2016. Large-volume immunomagnetic separation combined with multiplex PCR assay for simultaneous detection of Listeria monocytogenes and Listeria ivanovii in lettuce. Food Control 59:601608.

Martinez-Blanch, J. F., G. Sanchez, E. Garay, and R. Aznar. 2010. Evaluation of a real-time PCR assay for the detection and quan- 
tification of Bacillus cereus group spores in food. J. Food Prot. $73: 1480-1485$

Martínez-Blanch, J. F., G. Sánchez, E. Garay, and R. Aznar. 2011. Detection and quantification of viable Bacillus cereus in food by RT-qPCR. Eur. Food Res. Technol. 232:951-955.

Porcellato, D., J. Narvhus, and S. B. Skeie. 2016. Detection and quantification of Bacillus cereus group in milk by droplet digital PCR. J. Microbiol. Methods 127:1-6.

Priha, O., K. Hallamaa, M. Saarela, and L. Raaska. 2004. Detection of Bacillus cereus group bacteria from cardboard and paper with real-time PCR. J. Ind. Microbiol. Biotechnol. 31:161-169.

Shiota, M., K. Saitou, H. Mizumoto, M. Matsusaka, N. Agata, M. Nakayama, M. Kage, S. Tatsumi, A. Okamoto, S. Yamaguchi, M. Ohta, and D. Hata. 2010. Rapid detoxification of cereulide in $B a-$ cillus cereus food poisoning. Pediatrics 125: e951-e955.

Xing, X. J., W. Xiao, X. Liu, Y. Zhou, D. Pang, and H. Tang. 2016. A fluorescent aptasensor using double-stranded DNA/graphene oxide as the indicator probe. Biosens. Bioelectron. 78:431-437.

Xu, J., Y. Wang, L. Yang, Y. Gao, B. Li, and Y. Jin. 2017. A cytometric assay for ultrasensitive and robust detection of human telomerase RNA based on toehold strand displacement. Biosens. Bioelectron. 87:1071-1076.

Yu, B., F. Li, T. Zhao, F. Li, B. Zhou, and H. Xu. 2018. Hybridization chain reaction-based flow cytometric bead sensor for the detection of emetic Bacillus cereus in milk. Sens. Actuator B-Chem. 256:624-631.

Zhang, Y., Y. Liu, S. Zhen, and C. Huang. 2011. Graphene oxide as an efficient signal-to-background enhancer for DNA detection with a long range resonance energy transfer strategy. Chem. Commun. (Camb.) 47:11718-11720.

Zheng, A. X., J. Li, J. Wang, X. Song, G. Chen, and H. Yang. 2012. Enzyme-free signal amplification in the DNAzyme sensor via target-catalyzed hairpin assembly. Chem. Commun. (Camb.) 48:3112-3114.

Zhou, B., T. Liang, Z. Zhan, R. Liu, F. Li, and H. Xu. 2017. Rapid and simultaneous quantification of viable Escherichia coli O157: H7 and Salmonella spp. in milk through multiplex real-time PCR. J. Dairy Sci. 100:8804-8813. 